
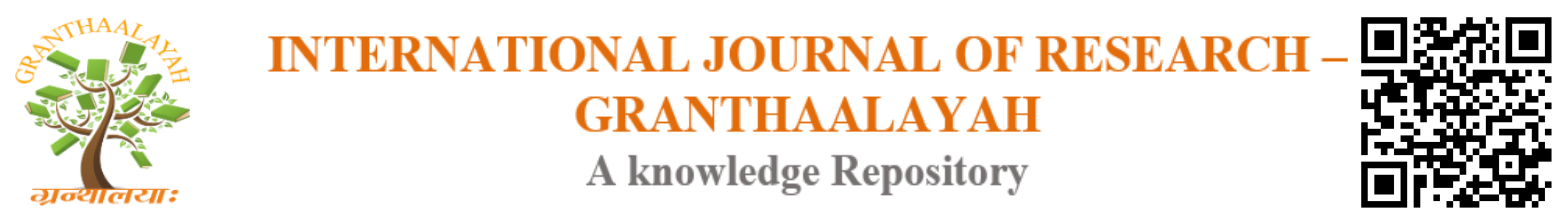

Science

\title{
FITTING OF AGE-SPECIFIC MORTALITY
}

\author{
A.S. Talawar ${ }^{* 1}$, Rajani P. Agadi ${ }^{2}$ \\ ${ }^{*} 1,2$ Department of Statistics, Karnatak University, Dharwad, India
}

\begin{abstract}
The age-pattern of mortality can be represented by various parametric models. In the present paper we consider a mixture of Weibull, Inverse-Weibull, and Gompertz-Makeham (GoMa) survival functions and Heligman-Pollard model to fit U.S. life table 2014. We use loss criterion for parameter estimation and demonstrate fitting of model. Both mixture and Heligman-Pollard model fit the mortality pattern reasonably well up to age 90 . We notice that the estimated mortality rates fit the actual pattern fairly well, although the fit at the earlier ages could be better. We have obtained the plots using our estimated values. The plots for mortality pattern of total population and other demographic characteristics (sex and race) are also considered.
\end{abstract}

Keywords: Force of Mortality; Survival Function; Loss Function; Life Table; HP Model.

Cite This Article: A.S. Talawar, and Rajani P. Agadi. (2019). "FITTING OF AGE-SPECIFIC MORTALITY." International Journal of Research - Granthaalayah, 7(7), 405-415. https://doi.org/10.29121/granthaalayah.v7.i7.2019.794.

\section{Introduction}

The laws of mortality attempt to summarize empirical observations and then used to construct life tables for human populations. They have been intimately linked with the statistical techniques of analyzing mortality data and described as parametric forms for quantities appearing in statistical models. A typical modern pattern of mortality can be divided into three periods. In the first period the mortality of infants, where in a rapid decrease of mortality is noticed during the first few years of life. The second period covers the young-adult mortality so-called accident hump where the deaths are mainly due to accidents. The third period indicates geometric increase of mortality with age (the rate of increase slowing down after age 80) called senescent mortality. Booth and Tickle [1] reviewed various methods of mortality modelling and forecasting of age-specific mortality rates.

In this paper we present a general law of mortality that is equal to a mixture of Weibull, InverseWeibull, and Gompertz - Makeham (GoMa) survival functions. We demonstrate a special case of our model that fits the pattern of mortality of a U.S. life table (by sex and race) up to age 90 . We apply loss criterion for parameter estimation including some information theoretic criterion. Plots based on our estimates of the mortality rates reasonably agree with [2, 3, 4]. Gompertz's law fits 
observed mortality rates very well at the adult ages, and it is a good tool for comparing mortality tables, as [4] demonstrated. Brillinger [5] argued that the distribution of the time of death can be approximated with one of the three extreme-value distribution functions. Carriere [2] also uses extreme-value distributions for modelling the pattern of mortality (for earlier literature see [6, 7 , $8,9])$.

The age-pattern of mortality can be represented by various parametric models. In particular, the Heligman-Pollard law takes into account, of three terms, infant mortality, young-adult mortality (accident hump), and senescent mortality. Heligman and Pollard [3] proposed a formula that fits Australian mortality rates fairly well at all ages. The first Heligman- Pollard law is given by the following expression

$q_{x} / p_{x}=A^{(x+B)^{c}}+D \exp \left\{-\exp (\log x-\log F)^{2}\right\}+G H^{x}$

Where the quantities $\left(q_{x} / p_{x}\right)$ are called the mortality odds, $p_{x}=1-q_{x}$ and $q_{x}$ is the probability that a life aged $x$ will die within a year. The Heligman and Pollard model is an eightparameter model containing three terms, each represents a distinct component of mortality. The last term reflects the exponential pattern of mortality at the adult ages, where as the first term reflects the decrease in mortality during childhood. The middle term models, the accident hump, at age 23 are found in many mortality tables.

According to HP1 $\ln \left(q_{x} / p_{x}\right)$ becomes a straight line with advancing age. Modifications have been brought for HP1 to bring improvements in the fit, especially at the older ages [2]. The modifications are;

$$
\begin{aligned}
& q_{x} / p_{x}=A^{(x+B)^{c}}+D \exp \left\{-\exp (\log x-\log F)^{2}\right\}+G H^{x} /\left(1+G H^{x}\right) \\
& q_{x} / p_{x}=A^{(x+B)^{c}}+D \exp \left\{-\exp (\log x-\log F)^{2}\right\}+G H^{x} /\left(1+K G H^{x}\right) \\
& q_{x} / p_{x}=A^{(x+B)^{c}}+D \exp \left\{-\exp (\log x-\log F)^{2}\right\}+G H^{x} /\left(1+G H^{x^{K}}\right)
\end{aligned}
$$

For recent applications of mortality laws, see [10, 11, 12, 13, 14, 15, 16]. Sharrow [17] investigates the sex-age-specific changes in the mortality of a prospectively monitored rural population in South Africa. They quantify changes in the age pattern of mortality efficiently by estimating the eight parameters of the Heligman-Pollard (HP1) model of age-specific mortality.

Section 2 deals with the conditional probability of survival. We present a general law of mortality that is equal to a mixture of Weibull, Inverse-Weibull, and Gompertz - Makeham (GoMa) survival functions in section 3. Section 4 gives results and discussion where in we consider the application of parametric mixture model and Heligman-Pollard model to life table data of U.S. population. Section 5 provides conclusion of the findings. 


\section{Conditional Probability of Survival}

The mortality table provides the probability of death or the probability of survival within any one given year, but the conditional probability of survival goes a step further. That is: if an individual is currently aged $x$, then the probability of surviving $n$ more years is defined by

$$
(n P x)=\prod_{i-0}^{n-1}\left(1-q_{x+i}\right)
$$

As long as $(t P x)$ is constant or decreasing with respect to $t$, then this function can be represented as

$$
(t P x)=\exp \left\{-\int_{x}^{x+t} \mu(s) d s\right\}
$$

Where $\mu(s) \geq 0$ for all $s \geq 0$ and $\mu(s)$ is the instantaneous rate of death at age $s$ or force of mortality. Note that by a simple change of variables $u=s-x$ we can rewrite the above equation as

$$
(t P x)=\exp \left\{-\int_{0}^{t} \mu(x+u) d u\right\}
$$

Therefore, the product of derivative of the cumulative distribution function $F_{x}(t)$ or $1-(t P x)$ and the force of mortality $\mu(x+t)$ is the probability density function $f_{x}(t)$ which is equivalent to

$f_{x}(t)=(1-(t P x)) \mu(x+t)$

\section{Mixtures of Parametric Survival Functions}

In this section we present a general law of mortality that is equal to a mixture of Weibull, InverseWeibull and Gompertz-Makeham (GoMa) survival functions. Carriere [2] argues that extremevalue survival functions are reasonable models for $s_{k}(x)$, specific cause $\mathrm{k}$ and then $s(x)=$ $\sum_{k=1}^{m} \varphi_{k} s_{k}(x)$. Thus, he presents the survival functions for the Gompertz, Weibull, InverseGompertz, and Inverse-Weibull models and also gives parameterization of these mixtures of models that reveal the location and dispersion of the distribution. Gompertz's law fits observed mortality rates very well at adult ages, and it is a good tool for comparing mortality tables. For certain parameter values the Weibull model has a decreasing force of mortality, and therefore he considers this model for early childhood.

\section{(a) GoMa Law of Mortality Model}

As in the case of the exponential law of mortality, the Gompertz and then the GoMa law of mortality derived using the instantaneous force of mortality (IFM) curve $\mu_{x}$

$\mu_{x}=\frac{1}{\sigma} \exp \left\{\frac{x-m}{\sigma}\right\}$

where $m>0$ is a measure of location because it is the modal value of life and $\sigma>0$ represents dispersion parameter of the density about this mode. In the GoMa case, the modal is defined as: 
$\mu_{x}=\lambda+\frac{1}{\sigma} \exp \left\{\frac{x-m}{\sigma}\right\}$

According to (1), the instantaneous force of mortality is a constant $\lambda$ plus a time dependent exponential curve. The constant $\lambda$ aims to capture the component of the death rate that is attributable to accidents, while the exponentially increasing portion reflects natural death causes. This curve increases with age and goes to infinity as $t \rightarrow \infty$. When the individual is exactly $x=m$ years old, the GoMa-IFM curve is $\mu(m)=\lambda+1 / \sigma$, but when the individual is younger $(x<$ $m)$ the GoMa- IFM curve is $\mu(x)<\lambda+1 / \sigma$, and when the individual is older $(x>m)$ the GoMa-IFM curve is $\mu(x)>\lambda+1 / \sigma$. Thus, $x=m$ is a special age point on the IFM curve, it is the modal value.

The conditional probability of survival under the GoMa-IFM curve is equal to

$(t P x)=\exp \left\{-\lambda t+\sigma(\mu(x)-\lambda)\left(1-e^{t / \sigma}\right)\right\}$

Notice that how the probability of survival declines, in time, at a rate faster than $\lambda$. The additional terms in the exponent are less than zero and thus accelerate the decline.

GoMa survival function for single year age is defined as

$s(x)=\exp \left\{\lambda t+e^{-m / \sigma}-e^{(x-m) / \sigma}\right\}$

(a) The Weibull Model

Another survival function is Weibull, which can be parameterized as follows

$s(x)=\exp \left\{-\left(\frac{x}{m}\right)^{m / \sigma}\right\}$

(b) The Inverse-Weibull Model

The Inverse-Weibull survival function is

$s(x)=1-\exp \left\{-\left(\frac{x}{m}\right)^{-m / \sigma}\right\}$

For details of the Weibull Model, Inverse-Weibull Model and their parameterization see [2].

The pattern of mortality suggests that a mixture of a Weibull, an Inverse-Weibull and a GoMa may be quite reasonable. In this case a survival function would have the form

$s(x)=\varphi_{1} s_{1}(x)+\varphi_{2} s_{2}(x)+\varphi_{3} s_{3}(x)$

Where $\varphi_{3}=1-\varphi_{1}-\varphi_{2}$

$s_{1}(x)=\exp \left\{-\left(\frac{x}{m_{1}}\right)^{m_{1} / \sigma_{1}}\right\}$

$s_{2}(x)=1-\exp \left\{-\left(\frac{x}{m_{2}}\right)^{-m_{2} / \sigma_{2}}\right\}$

$s_{3}(x)=\exp \left\{\lambda t+e^{-m_{3} / \sigma_{3}}-e^{-\left(x-m_{3}\right) / \sigma_{3}}\right\}$ 
For instance, $\varphi_{1}$ is the probability that a new life will die of childhood causes; $\varphi_{2}$ is the probability of dying of teenage causes; and $\varphi_{3}$ is the probability of dying of adult causes. Moreover, the location $(\mathrm{m})$ and scale parameters $(\sigma)$ provide some useful statistical information. We estimate the parameters $\varphi_{k}, m_{k}, \sigma_{k}$ for $\mathrm{k}=1,2,3$, such that the loss function (11) is minimum. All parameter estimates are calculated by statistical computer program called R-programming. Therefore, this system estimated the parameters by minimizing

$$
\begin{aligned}
& \sum_{x=0}^{90}\left(1-\hat{q}_{x} / q_{x}\right)^{2} \\
& \sum_{x=0}^{90}\left(1-\hat{d}_{x} / d_{x}\right)^{2}
\end{aligned}
$$

where $\hat{q}_{x}$ is fitted value at age $\mathrm{x}$ and $q_{x}$ is the observed mortality rate. That is, the sum of the squares of the proportional difference between the fitted and observed rates is minimized. The observed rates above age 85 are excluded from the calculation because they appeared to be less reliable.

In these formulae $q_{x}$ is a U.S. mortality rate and $d_{x}=s(x) q_{x}$ is the probability that a new life will die between the ages of $x$ and $x+1$. Estimates of $q_{x}$ and $d_{x}$ are

$\hat{q}_{x}=1-\hat{s}(x / 1) / \hat{s}(x)$

$\hat{d}_{x}=\hat{s}(x)-\hat{s}(x+1)$

Where $\widehat{s}(x)$ is equal to (7-10) evaluated at the estimated parameters.

\section{Results and Discussion}

We consider an application of mixture model and Heligman-Pollard model to a life table for the total, male, female, black and white population of the U.S. and loss criterion for parameter estimation. The values of parameters for the mixture model are presented in Table 1. The U.S. Life Tables for 2014 based on 2010 decennial census and 2014 Medicare data for persons aged 66-69 were prepared by the Division of Vital Statistics [18]. The pattern of mortality can be described by the function $\log \left(q_{x}\right)$. The "Fig. (a-i)" illustrate mixture of Weibull, Inverse-Weibull and GoMa survival functions and "Fig. (j-r)" illustrate Heligman-Pollard model for the total male \& female and black \& white U.S. population at the ages $x=0, \ldots, 90$. These graphs are very informative because we can immediately identify at least three components to the pattern of mortality. First, $\log \left(q_{x}\right)$ is decreasing at the early ages. Second, there appears to be a hump at around age 23. Third, there is a linear component at the adult ages. The mortality patterns in "Fig. (a-i)" suggest that a mixture of a Weibull, an Inverse-Weibull and a GoMa may be reasonable. From the "Fig. (a-i)" and "Fig. (j-r)", we notice that the estimated mortality rates fit the actual pattern fairly well, although the fit at the earlier ages could be better. The location parameters $m_{1}, m_{2}$, and $m_{3}$ clearly show that the Weibull component models the early ages, the Inverse-Weibull component models the ages from 10 to 35 and the GoMa component models the late ages. The parameter $\varphi_{3}$ reveals that the GoMa component explains most of the deaths in the U.S. population. 
The observed and fitted mortality rates for U. S. total, male, female, black and white population of 2014 are presented graphically in "Fig. (a-i)" and "Fig. (j-r)". The graduated mortality probabilities appear to provide adequate representations of the age patterns of mortality in all nine cases whether or not the accidents hump is strong and prominent although a few minor defects are evident. Where the accident hump is prominent, the fitting appears to overstate mortality on part of the downward portion of the curve and understate it for a few years thereafter. The parameters estimated using loss function for the "Fig. (a - i)" are presented in Table 1.

The parameters estimated for the "Fig. ( $\mathrm{j}-\mathrm{r})$ " are presented in Table 2. Parameters A, B and C describe the pattern of mortality during early childhood. The value of $\mathrm{A}$ is more in female population as compared to male population. It indicates that female child mortality is more when compared to that of males. Therefore, different population females have experienced higher child mortality than males (parameter A). The drop in mortality after age 1 , indicated by $\mathrm{C}$. In other words, mortality has been declining faster in recent years at the younger age than at the older childhood ages. For both males and females the parameter B has also been falling. Female child mortality is low and also its rate declines with age faster throughout the year period (females have higher values of $\mathrm{C}$ ). The higher values of $\mathrm{B}$ for females indicate that females had relatively lower infant mortality.

Accident mortality is now concentrated in a narrower band of ages, and as a result $\mathrm{E}$ has increased. The location of the hump however has remained more or less constant near age 25 (parameter F). When we compare the accident hump for males and females we see that males experienced slightly more mortality at accident hump (parameter D). The parameter value E for male (white and black) at hump is less as compared to female (white and black). The location of the accident hump (F) for females is at a slightly younger age than that for males. The third term in (HP1) represents the ageing of the body (senescent mortality), and its parameters ( $\mathrm{G}$ and $\mathrm{H}$ ) describe the age pattern of mortality at the older ages. It is clear that with advancing age $\log \left(q_{x}\right)$ approaches the straight line. The base senescent parameter $\mathrm{G}$ for males (total, white and black population) is more compared to female population (total, white and black), indicating higher male mortality throughout the senescent age span. The other parameter $\mathrm{H}$ of senescent period more or less remains the same for males and females (total, white and black population).
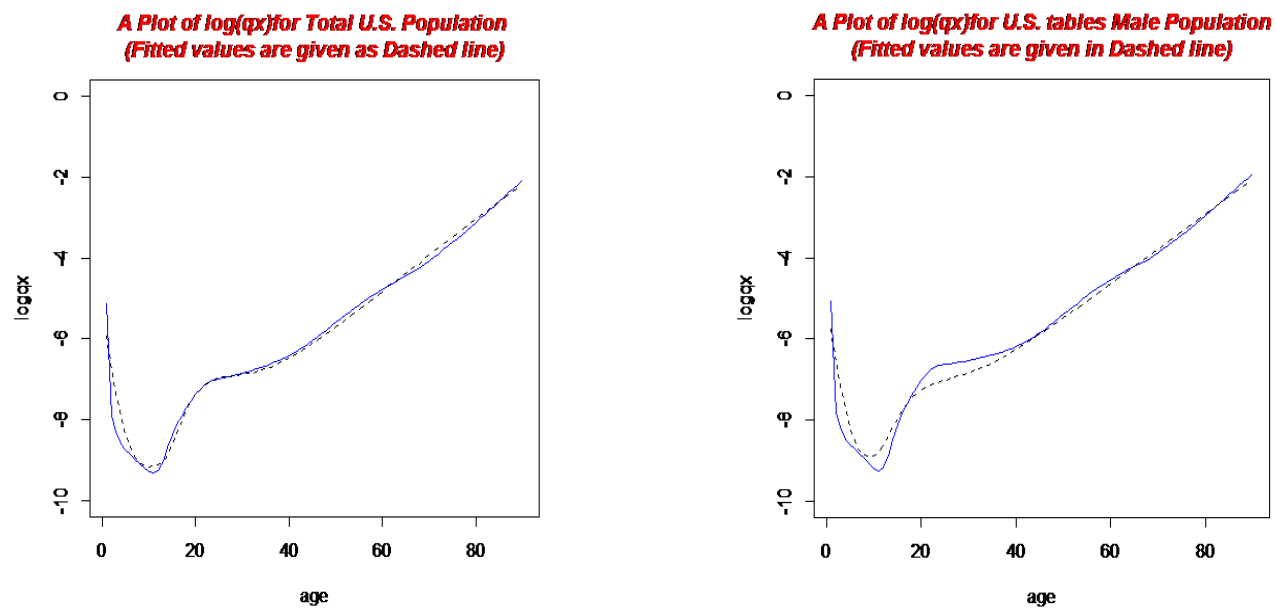

Figure (a-b): Fitting of age-specific mortality rates of total and males population for mixed Weibull, Inverse-Weibull and GoMa Survival functions using U S life tables 2014 

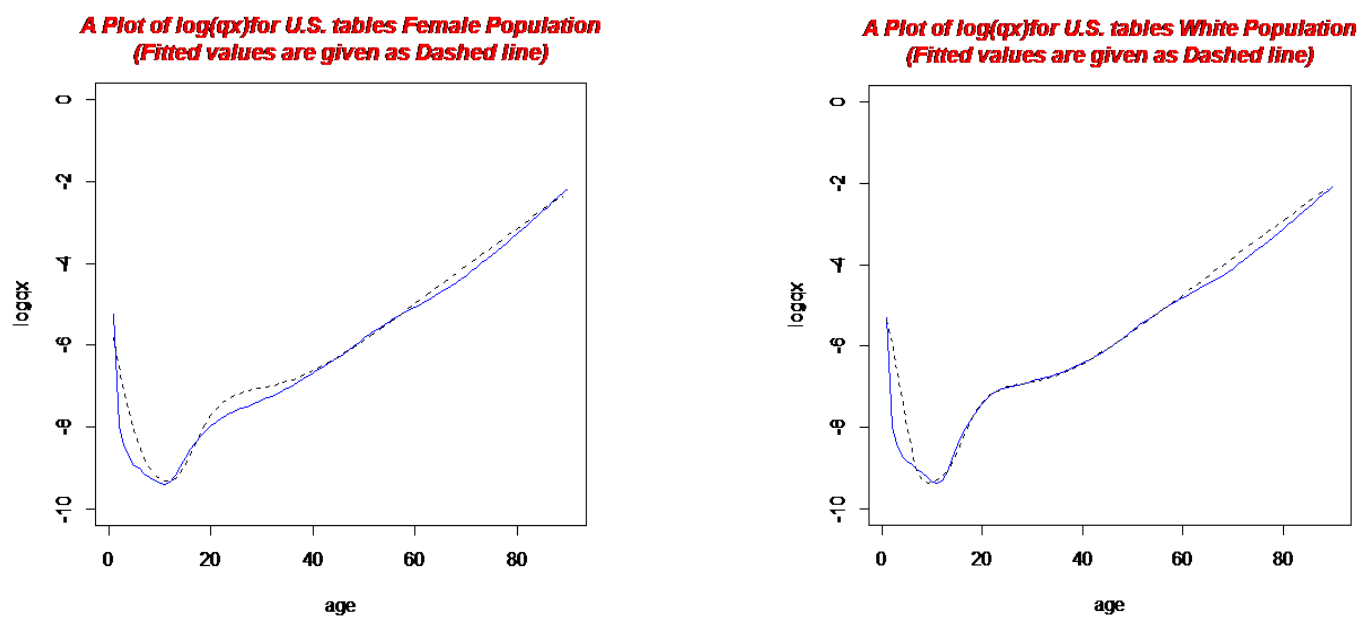

Figure (c-d): Fitting of age-specific mortality rates of females and white population for mixed Weibull, Inverse-Weibull and GoMa Survival functions using U S life tables 2014
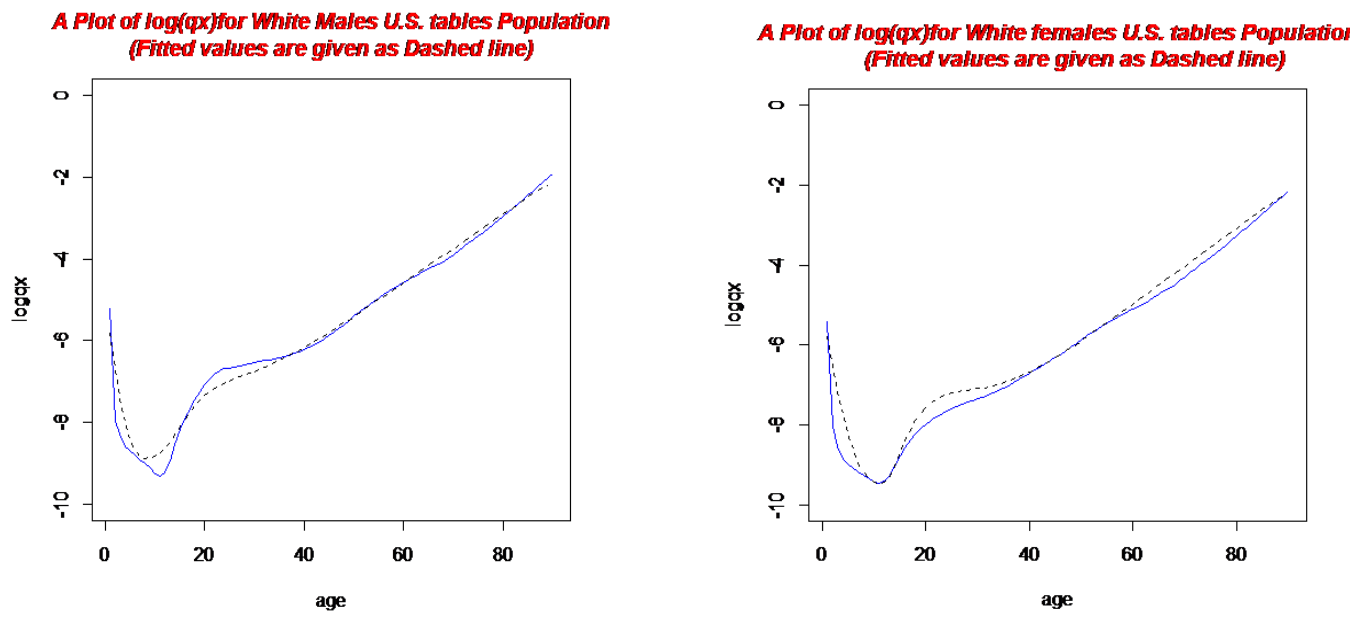

Figure (e-f): Fitting of age-specific mortality rates of white males and females population for mixed Weibull, Inverse-Weibull and GoMa Survival functions using U S life tables 2014
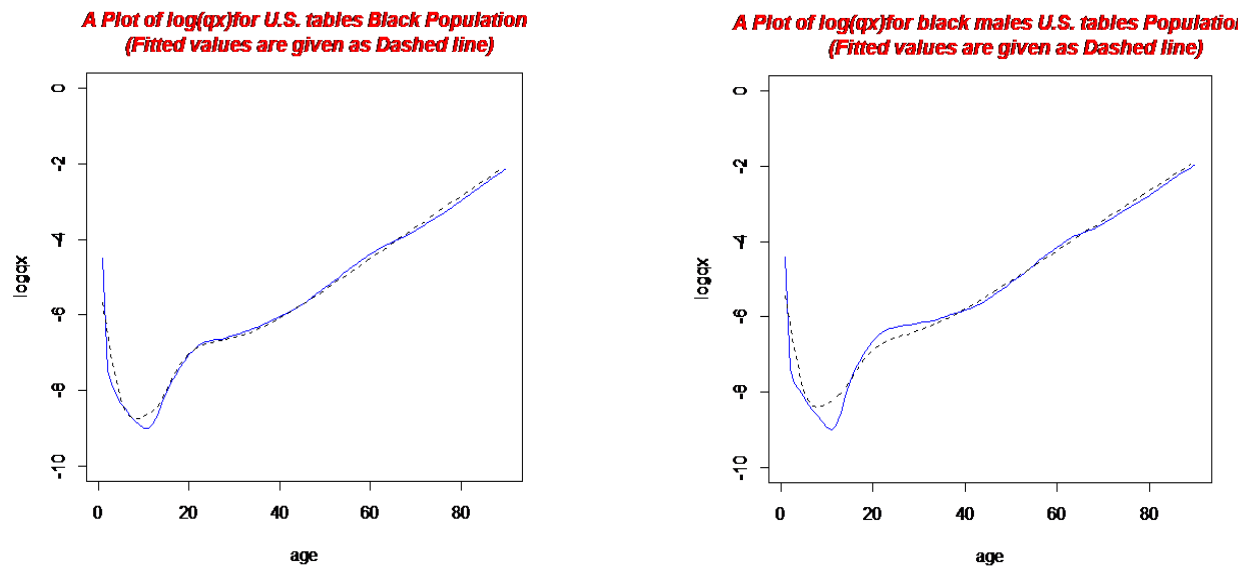

Figure (g-h): Fitting of age-specific mortality rates of black total \& males population for mixed

Weibull, Inverse-Weibull and GoMa Survival functions using U S life tables 2014 

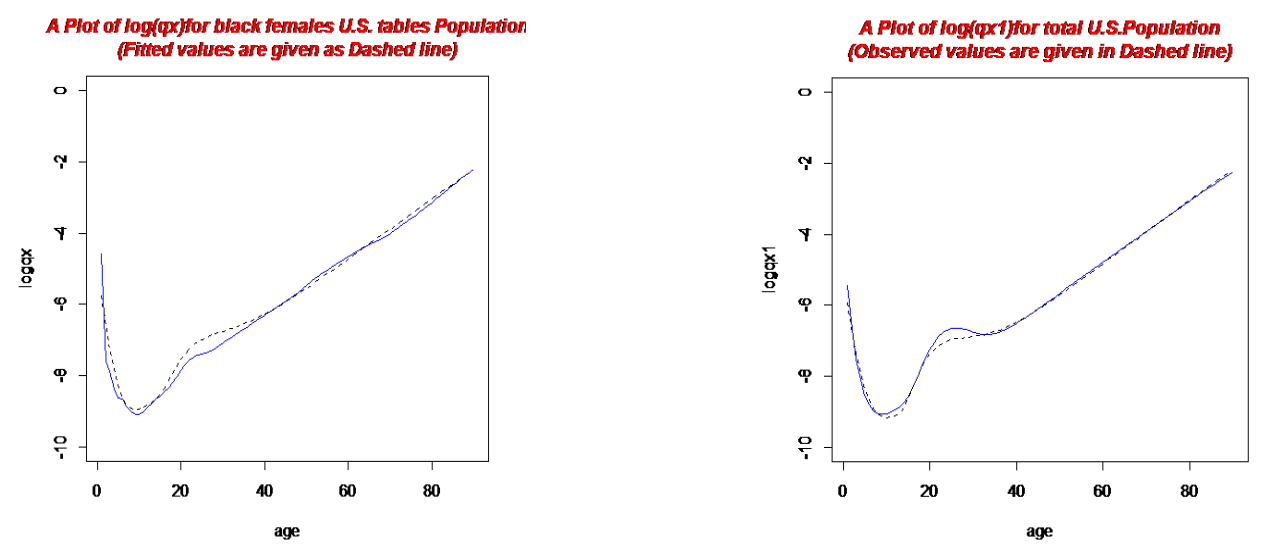

Figure (i-j): Fitting of age-specific mortality rates of black female for mixed Weibull, InverseWeibull and GoMa Survival functions and total population for Heligman-Pollard Model using U S life tables 2014
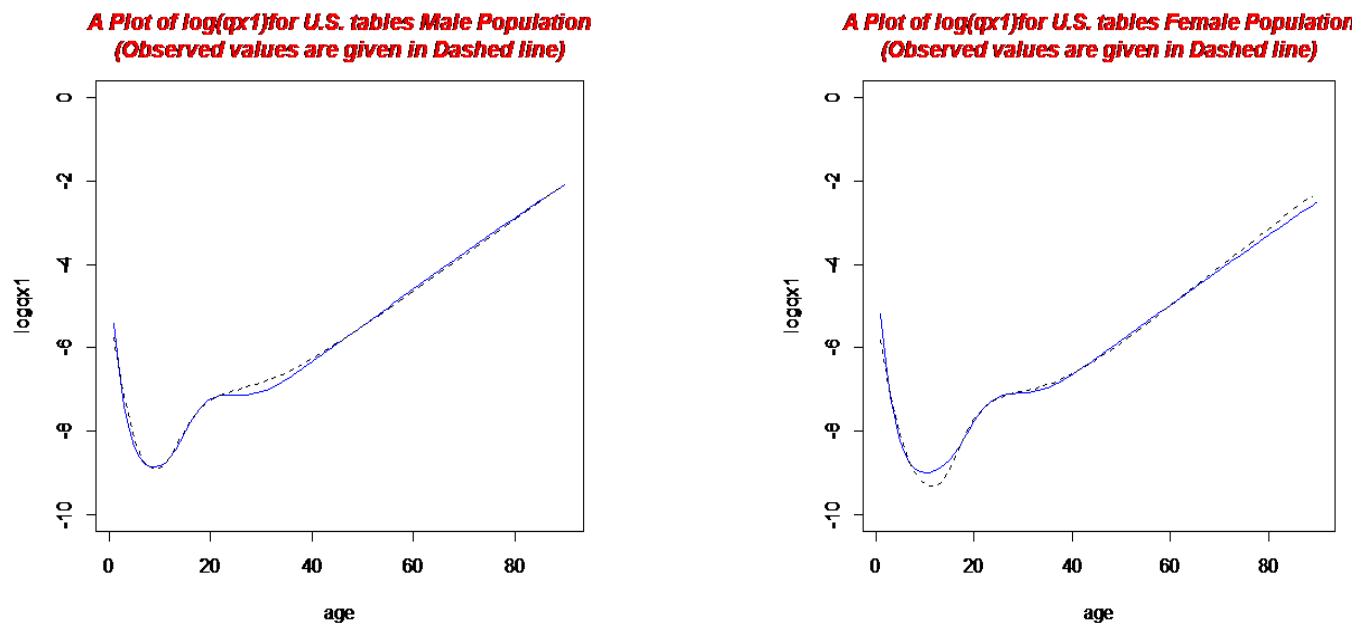

Figure (k-1): Fitting of age-specific mortality rates of white males and females population for Heligman-Pollard Model using U S life tables 2014
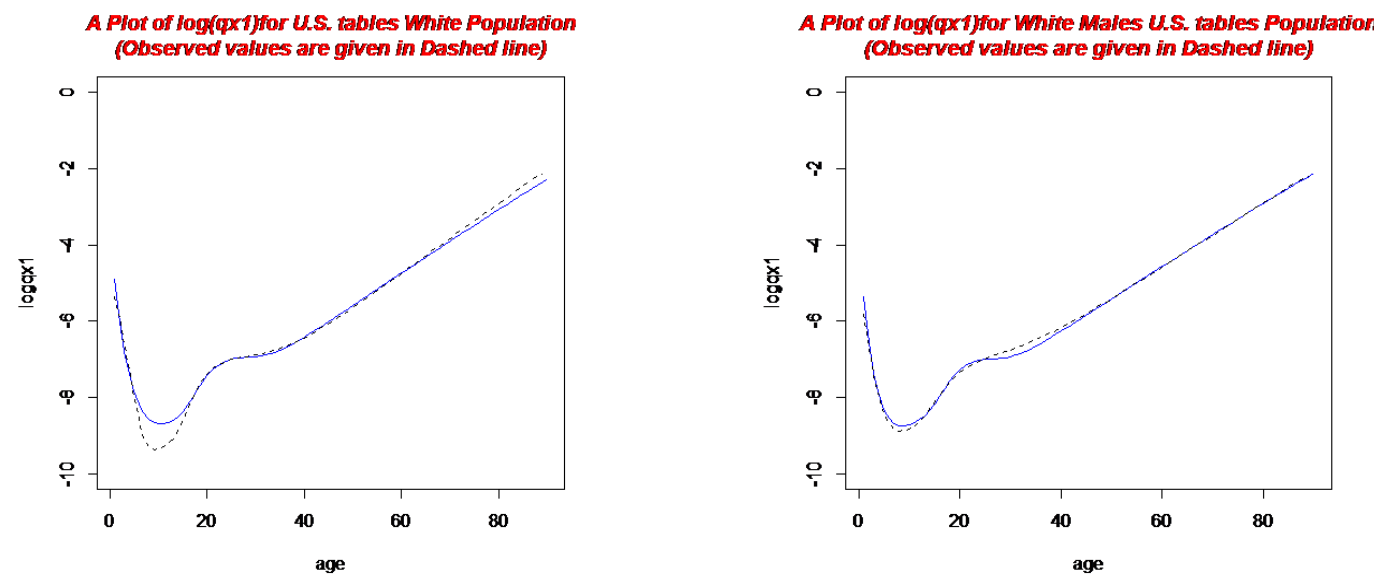

Figure (m-n): Fitting of age-specific mortality rates of white total and males population for Heligman-Pollard Model using U S life tables 2014 

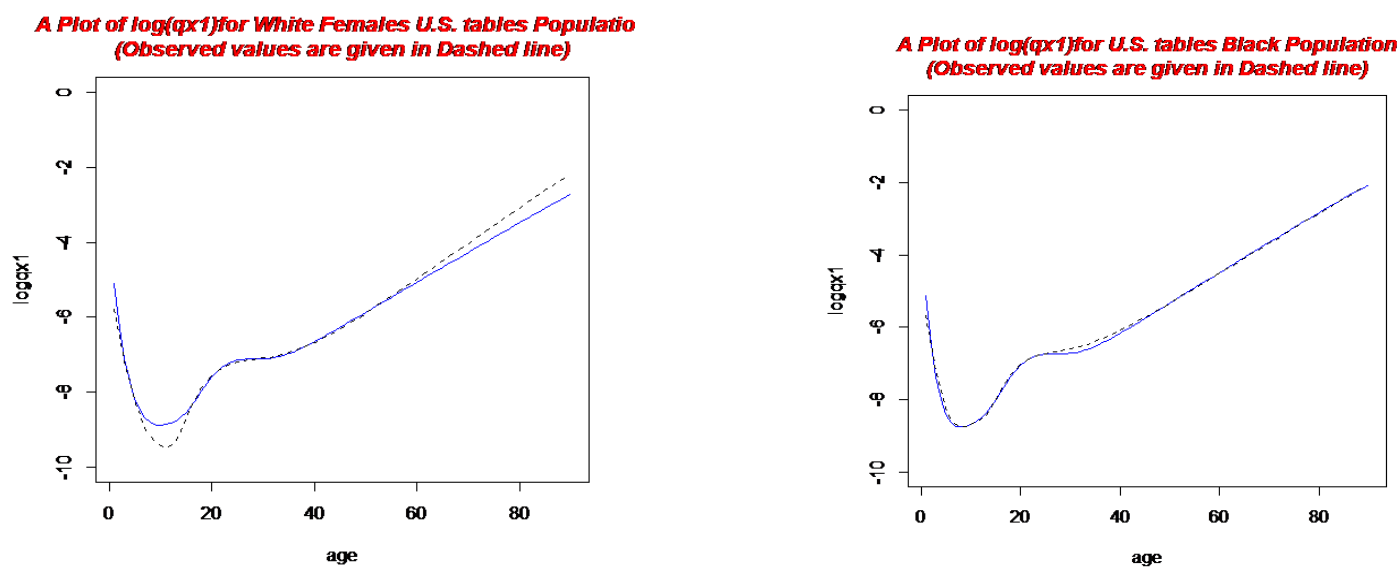

Figure (o-p): Fitting of age-specific mortality rates of white females and black population for Heligman-Pollard Model using U S life tables 2014
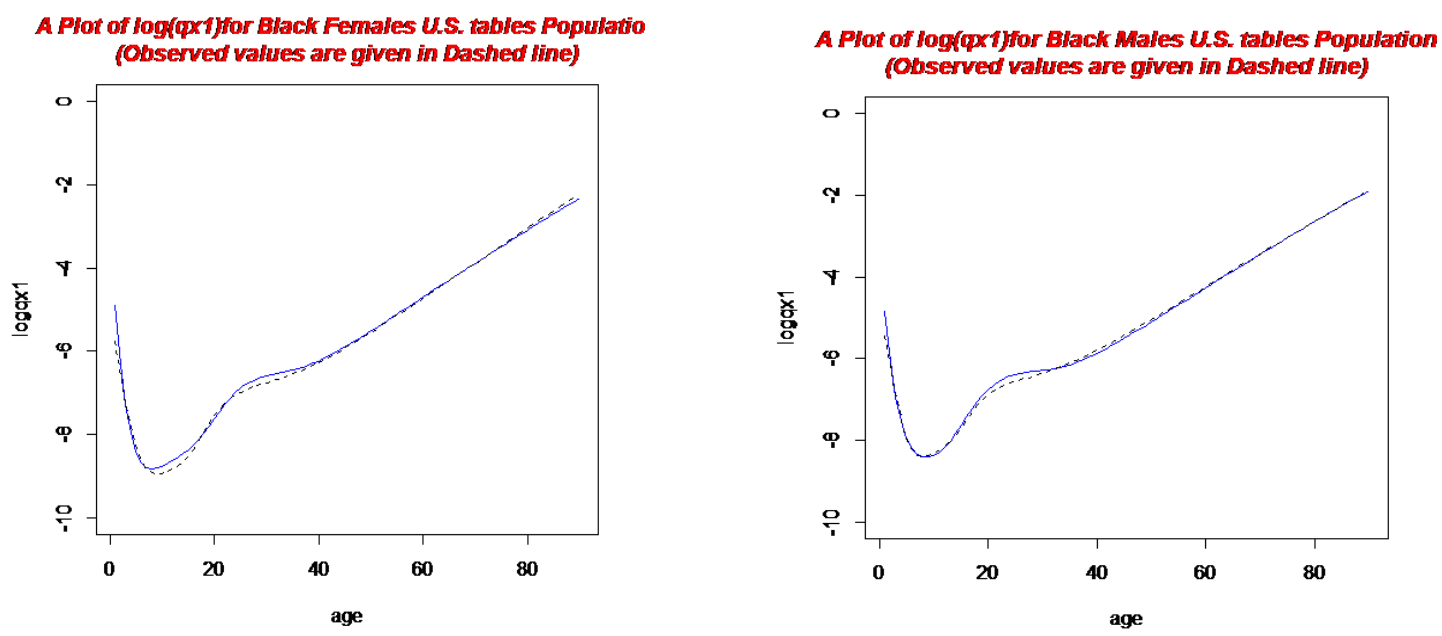

Figure (q-r): Fitting of age-specific mortality rates of white total, males \& females and black population for Heligman-Pollard Model using U S life tables 2014

\section{Conclusion}

The mixture of Weibull, Inverse- Weibull, and GoMa survival functions gives a good fit to mortality pattern of U. S. Life table 2014 for the total population, male \& female and, black \& white population. Heligman - Pollard (HP) model also gives good fit to the mortality data. The GoMa law explains most of the patterns of mortality (as the third part of HP model explains) in all the analyzed tables. In particular the following aspects can be pointed out, i. a decrease in the infant mortality, and in one-year probabilities of dying, $q_{x}$ in particular at adult and old ages, ii. an increasing concentration of deaths around the mode at old ages (that is, the Lexis point) of the curve of deaths and iii. higher levels and a larger dispersion of accidental deaths at young-adult ages (the so-called young mortality hump). 
Table 1: Estimated parameters values for mixed Weibull, Inverse-Weibull and GoMa Survival functions Using U S Life Table 2014

\begin{tabular}{|c|c|c|c|c|c|c|c|c|c|}
\hline \multirow{2}{*}{ Parameter } & \multicolumn{3}{|c|}{ Total Population } & \multicolumn{3}{c|}{ White Population } & \multicolumn{3}{c|}{ Black Population } \\
\cline { 2 - 10 } & Total & Male & Female & Total & Male & Female & Total & Male & Female \\
\hline$\varphi_{1}$ & 0.01632 & 0.01632 & 0.01632 & 0.01632 & 0.01632 & 0.01632 & 0.01632 & 0.01632 & 0.01632 \\
\hline$m_{1}$ & 0.7962 & 0.9958 & 1.08 & 1.7735 & 0.8365 & 1.03 & 1.01 & 1.33 & 0.9607 \\
\hline$\sigma_{1}$ & 1.17 & 1.28 & 1.48 & 1.49 & 1.07 & 1.38 & 1.18 & 1.32 & 1.21 \\
\hline$\varphi_{2}$ & 0.01385 & 0.01385 & 0.1385 & 0.01385 & 0.01385 & 0.01385 & 0.01385 & 0.01385 & 0.01385 \\
\hline$m_{2}$ & 27.85 & 28.37 & 30.57 & 28.09 & 30.98 & 28.92 & 26.59 & 26.91 & 30.95 \\
\hline$\sigma_{2}$ & 9.07 & 12.61 & 11.18 & 9.57 & 13.68 & 10.91 & 8.75 & 9.18 & 10.69 \\
\hline$\varphi_{3}$ & 0.96983 & 0.96983 & 0.96983 & 0.96983 & 0.96983 & 0.96983 & 0.96983 & 0.96983 & 0.96983 \\
\hline$m_{3}$ & 87.34 & 85.64 & 88.63 & 86.05 & 85.38 & 87.98 & 84.68 & 81.76 & 87.05 \\
\hline$\sigma_{3}$ & 11.01 & 11.41 & 10.71 & 10.761 & 11.69 & 10.41 & 11.89 & 12.27 & 11.67 \\
\hline$\lambda$ & 8.9 & 9.7 & 5.1 & 7.9 & 6.3 & 6.2 & 4.9 & 7.1 & 8.3 \\
& $\times 10^{-7}$ & $\times 10^{-7}$ & $\times 10^{-9}$ & $\times 10^{-8}$ & $\times 10^{-8}$ & $\times 10^{-8}$ & $\times 10^{-8}$ & $\times 10^{-8}$ & $\times 10^{-8}$ \\
\hline
\end{tabular}

Table 2: Estimated parameters values for Heligman-Pollard Model Using U S Life Table 2014

\begin{tabular}{|c|c|c|c|c|c|c|c|c|c|}
\hline \multirow{2}{*}{3399} & \multicolumn{3}{|c|}{ Total Population } & \multicolumn{3}{c|}{ White Population } & \multicolumn{3}{c|}{ Black Population } \\
\cline { 2 - 10 } & Total & Male & Female & Total & Male & Female & Total & Male & Female \\
\hline A & 0.004964 & 0.005105 & 0.006434 & 0.008386 & 0.005371 & 0.006886 & 0.006886 & 0.00886 & 0.008086 \\
\hline B & 0.08348 & 0.08902 & 0.08748 & 0.08168 & 0.08968 & 0.08997 & 0.9297 & 0.08041 & 0.05018 \\
\hline C & 0.3218 & 0.3138 & 0.3217 & 0.3217 & 0.3217 & 0.3264 & 0.3664 & 0.3562 & 0.3862 \\
\hline D & 8.918 & 4.291 & 4.089 & 4.689 & 4.189 & 4.089 & 6.249 & 9.396 & 6.239 \\
& $\times 10^{-4}$ & $\times 10^{-4}$ & $\times 10^{-4}$ & $\times 10^{-4}$ & $\times 10^{-4}$ & $\times 10^{-4}$ & $\times 10^{-4}$ & $\times 10^{-4}$ & $\times 10^{-4}$ \\
\hline E & 13.3862 & 9.8862 & 12.3986 & 11.0686 & 12.0016 & 12.0686 & 10.0764 & 7.01 & 10.71 \\
\hline F & 24.91 & 21.19 & 25.69 & 24.52 & 22.71 & 24.52 & 23.36 & 24.8 & 28.8 \\
\hline G & 4.269 & 5.189 & 4.169 & 5.267 & 6.267 & 5.003 & 6.803 & 8.982 & 6.495 \\
& $\times 10^{-5}$ & $\times 10^{-5}$ & $\times 10^{-5}$ & $\times 10^{-5}$ & $\times 10^{-5}$ & $\times 10^{-5}$ & $\times 10^{-5}$ & $\times 10^{-5}$ & $\times 10^{-5}$ \\
\hline H & 1.092 & 1.092 & 1.089 & 1.089 & 1.089 & 1.084 & 1.089 & 1.088 & 1.086 \\
\hline
\end{tabular}

\section{References}

[1] Booth, H. and Tickle, L. Mortality Modelling and Forecasting: A Review of Methods. A.A.S. 3, I/II, 2008, 3-43.

[2] Carriere, J.F. Parametric models for life tables. Insurance: Mathematics and Economics, 14(1), 1992, 77-100.

[3] Heligman, L.M.A. and Pollard, J.H. The Age Pattern of Mortality, Journal of the Institute of Actuaries 107, part 1, 1980, 49-82.

[4] Wetterstrand, W.H. Parametric Models for Life Insurance Mortality Data: Gompertz's Law over Time, TSA XXXIII, 1981, 159-79.

[5] Brillinger, D.R. A Justification of Some Common Laws of Mortality, TSA XIII, Part 1, 1961, $116-26$.

[6] Jordan, C.W. Life Contingencies. Chicago, Ill.: Society of Actuaries, 1967.

[7] Tenenbmn, A. and Vanderhoof, I.T. New Mathematical Laws of Select and Ultimate Mortality, TSA, XXXII, 1980, 119-83.

[8] Siler, W. Parameters of mortality in human populations with widely varying life spans. Statistics in medicine, 2(3), 1983, 373-380.

[9] Hartmann, M. Past and recent attempts to model mortality at all ages. Journal of Official Statistics, 3(1), 1987, 19-36. 
[10] Debón, A., Montes, F. and Sala, R. A comparison of parametric models for mortality graduation: Application to mortality data for the Valencia region (Spain). SORT, 29(2), 2005, 269-288.

[11] Milevsky, M. A. The Calculus of Retirement Income: Financial Models for Pension Annuities and Life Insurance, Cambridge University Press, 2006.

[12] Bebbington, M., Lai, C.D. and Zitikis, R. Modeling human mortality using mixtures of bathtub shaped failure distributions, Journal of Theoretical Biology, 245(3),2007, 528-538.

[13] Smith, J., Mushati, P., Kurwa, F., Mason, P., Gregson, S. and Lopman, B. Changing patterns of adult mortality as the HIV epidemic matures in Manicaland, eastern Zimbabwe. AIDS, 21(6), 2007, 81-86.

[14] Zaba, B., Marston, M., Crampin, A.C., Isingo, R., Biraro, S., Bärnighausen, T., Lopman, B., Lutalo, T., Glynn, J.R. and Todd, J. Age-specific mortality patterns in HIV infected individuals: a comparative analysis of Africa community study data. AIDS, 21(6), 2007, 87-96.

[15] Milne, E.M.G. A New Model of Ageing and Mortality, B.A.J. 15, Supplement, 2009, 213-234.

[16] Pitacco, E. Health Insurance: basic actuarial models, Springer, International Publishing Switzerland, 2014.

[17] Sharrow, D., Clark, S., Collinson, M, Kahn, K. and Tollman. S. The Age Pattern of Increases in Mortality Affected by HIV: Bayesian Fit of the Heligman-Pollard Model to Data from the Agincourt HDSS Field Site in Rural Northeast South Africa. Demographic Research, 29, 2013, 1039-1096.

[18] NVSS. National Vital Statistics Reports, U S National Vital Statistics System, 66, No.4, 2017.

\footnotetext{
*Corresponding author.

E-mail address: talwarappu@ gmail.com/rajanipagadi2020@ gmail.com
} 